\title{
Erratum to: Models of Tumor Progression in Prostate Cancer
}

\author{
Waqas Azeem, Yaping Hua, Karl-Henning Kalland, Xisong Ke, \\ Jan Roger Olsen, Anne Margrete Øyan, and Yi Qu
}

\section{Erratum to:}

Chapter 19 in: L.A. Akslen and R.S. Watnick (eds.), Biomarkers of the Tumor Microenvironment, Basic Studies and Practical Applications DOI 10.1007/978-3-319-39147-2_19

Reference intext call out in chapter 19 was missed-out and the same has been updated in the chapter. 